\title{
Experience of designing a low-pressing turbocharger compressor using the modern version of a Universal modelling method
}

\author{
Yuri Galerkin ${ }^{1}$, Aleksandr Drozdov ${ }^{1, *}$, and Sergey Sibiriakov ${ }^{2}$ \\ ${ }^{1}$ Peter the Great St.Petersburg Polytechnic University St.Petersburg, Polytechnicheskaya, 29, Russia \\ ${ }^{2} \mathrm{NPO}$ "Turbotekhnika" Protvino, Zavodskoy passage, 4., Russia
}

\begin{abstract}
The paper presents the joint experience of the NPO "Turbotekhnika" and the R\&D Laboratory "Gas Dynamics of Turbomachines" of SPbPU for designing a centrifugal compressor operating at pressure ratio 1.61 and a mass flow rate of $0.62 \mathrm{~kg} / \mathrm{s}$. The design was executed using the Universal modelling method and the inviscid quasi-three-dimensional calculation program 3DM.023. At the first step, by the preliminary design program, the stage dimensions were determined. The expected gas-dynamic characteristics are calculated. At the final design step, stator elements were offered by NPO "Turbotekhnika" and the configuration of the impeller blades was optimized based on the non-viscous quasi-three-dimensional calculations. NPO "Turbotekhnika" designed, manufactured and tested the 140E compressor at blade velocities 150,200, 250 and $300 \mathrm{~m} / \mathrm{s}$. A comparison and analysis of experimental and calculated characteristics is presented. The design pressure ratio was calculated almost exactly for the design flow rate at a blade velocity of $300 \mathrm{~m} / \mathrm{s}$. The expected efficiency was confirmed. The mathematical model slightly overestimates the efficiency and the pressure ratio for the off-design flow rates.
\end{abstract}

\section{Introduction}

For optimal gas-dynamic design of centrifugal compressors, various approaches are used: CFD calculations, appropriate engineering mathematical models, etc. [1-4]. The CFD calculations of the turbocharger gas-dynamic characteristics are presented in paper [5]. The measured and calculated efficiency characteristics of a single-stage turbocharger TK23N-06 with a vaneless diffuser and a scroll are shown in Figure 1.

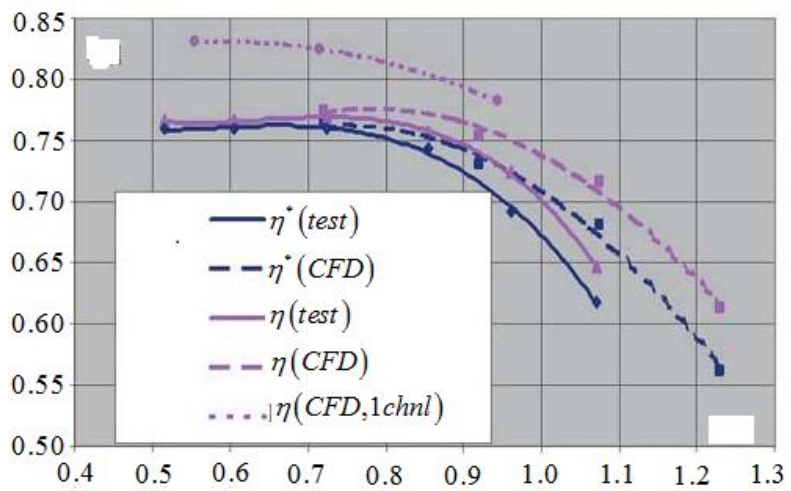

Fig. 1. The efficiency versus mass flow rate $(\mathrm{kg} / \mathrm{s})$ of the compressor TK23N-06 at $\mathrm{n}=21000 \mathrm{rpm}$ [5].

In the Figure 1: $\eta^{*}$ is efficiency depending on total parameters, $\eta$ is efficiency depending on static parameters, "chnl" has been calculated for one blade sector.

The maximal stage efficiencies calculated by the CFD more or less agree with the experimental data. But the calculated characteristic is biased towards high flow rates. The simulation is unsatisfactory.

The application of engineering mathematical models for calculating the turbocharger compressor is presented in paper [6]. The calculation is performed in a onedimensional setting. The flow parameters are determined in five control sections: at the impeller inlet, at the vaneless section inlet, at the vane diffuser inlet, at the scroll inlet and the exit from the stage. Figure 2 presents a comparison of experimental and TuCMS calculated performances. Calculated performances are significantly different from experimental. This indicates a lack of model elaboration and the need for its further development.

There are many other publications that demonstrate inefficiency of commercial CFD programs for centrifugal compressor characteristics simulation. The sample in Figure 2 demonstrates that correct engineering simulation is not easy, too.

\section{Aims and problems}

NPO "Turbotekhnika" works on the turbocharger for the $8 \mathrm{CN} 26 / 26$ gas engine. Preliminary matching analysis of the turbocharger and the engine has demonstrated that

\footnotetext{
*orresponding author: $\underline{\text { A drozdi@mail.ru }}$
} 

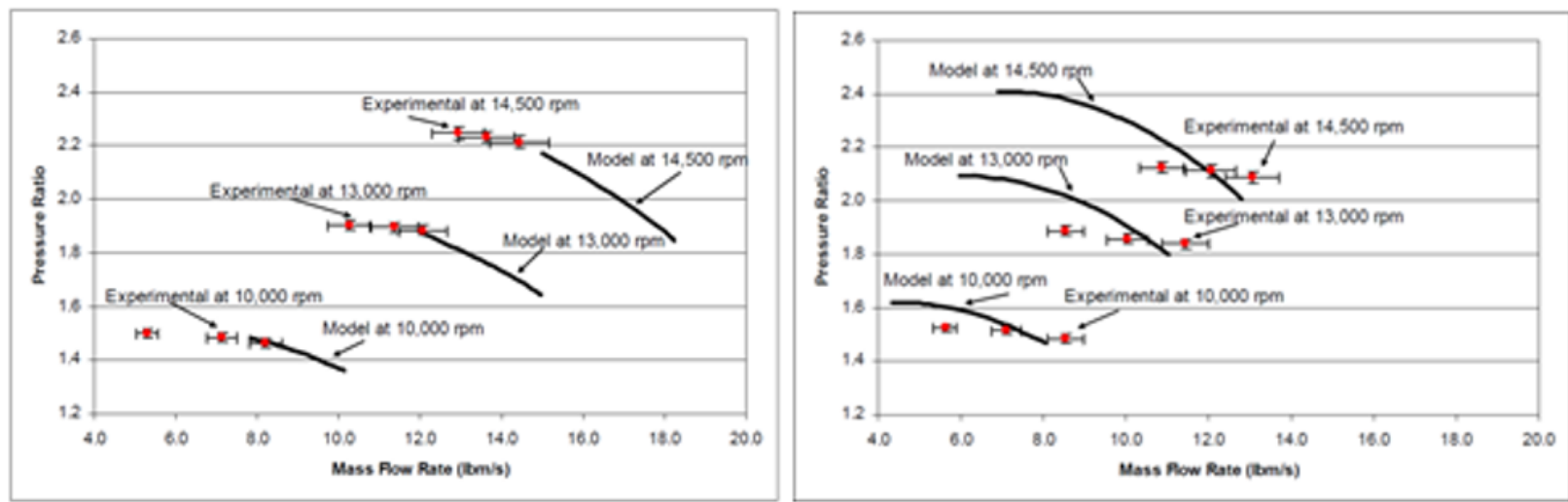

Fig. 2. Comparison of experimental characteristics and calculations performed using the program TuCMS [6].

the compressor pressure ratio at the design flow rate must be about 1.6. A large range of flow rate is needed.

In gas-dynamic design of the compressor the R\&D laboratory "Gas Dynamics of Turbomachines" (LGDTM) of SPbPU has applied its Universal modelling method [0-0]. It allows to perform end-to-end optimal design using the following steps:

- search for the optimal compressor version using a simplified mathematical model without the flow path dimensions;

- primary design, i.e., determining the basic dimensions of the flow path and the blade configuration, corresponding to a design flow rate and pressure ratio of the compressor;

- optimization and calculation of characteristics using the last (8-th) version of mathematical models of efficiency and work coefficient;

- digital description of the flow path and the formation of compressor flow path solid models.

Aim of the research is to design a low pressure ratio turbocharger compressor, to manufacture and test an experimental turbocharger. Evaluate the applicability of the Universal modelling method to effective design of turbocharger compressors.

\section{140E compressor parameters and candidates comparison}

Figure 3 (left) shows the menu of the candidates' comparison and preliminary design PC software with compressor parameters $\left(\bar{m}=0.62 \mathrm{~kg} / \mathrm{s}, \pi^{*}=p_{e x}^{*} / p_{i n l}^{*}=\right.$ 1.61 , where $\bar{m}$ is mass flow; $\pi^{*}$ is pressure ratio; $p_{\text {inl }}^{*}$ is inlet total pressure; $p_{e x}^{*}$ is exit total pressure). The turbine with $41000 \mathrm{rpm}$ was chosen by NPO "Turbotechnica" for the turbocharger. Figure 3 (right) shows the compressor candidates' efficiency, calculated using a simplified mathematical model [10-12].

The compared candidates have different loading factors $\psi_{T \text { des }}=c_{u 2} / u_{2}$ (where $c_{u 2}$ is the circumferential velocity component; $u_{2}$ is blade velocity; $\psi_{\text {Tdes }}$ is the design loading factor).

The bigger $\psi_{T \text { des }}$ is, the smaller is an impeller diameter and bigger is flow rate coefficient $\Phi_{\text {des }}=\frac{\bar{m}_{\text {des }}}{\rho_{\text {inl }}^{*} \cdot 0,785 D_{2}^{2} u_{2}} \quad\left(\bar{m}_{\text {des }}\right.$ is design mass flow rate, $\rho_{\text {inl }}^{*}$ is gas density depending on total parameters at an
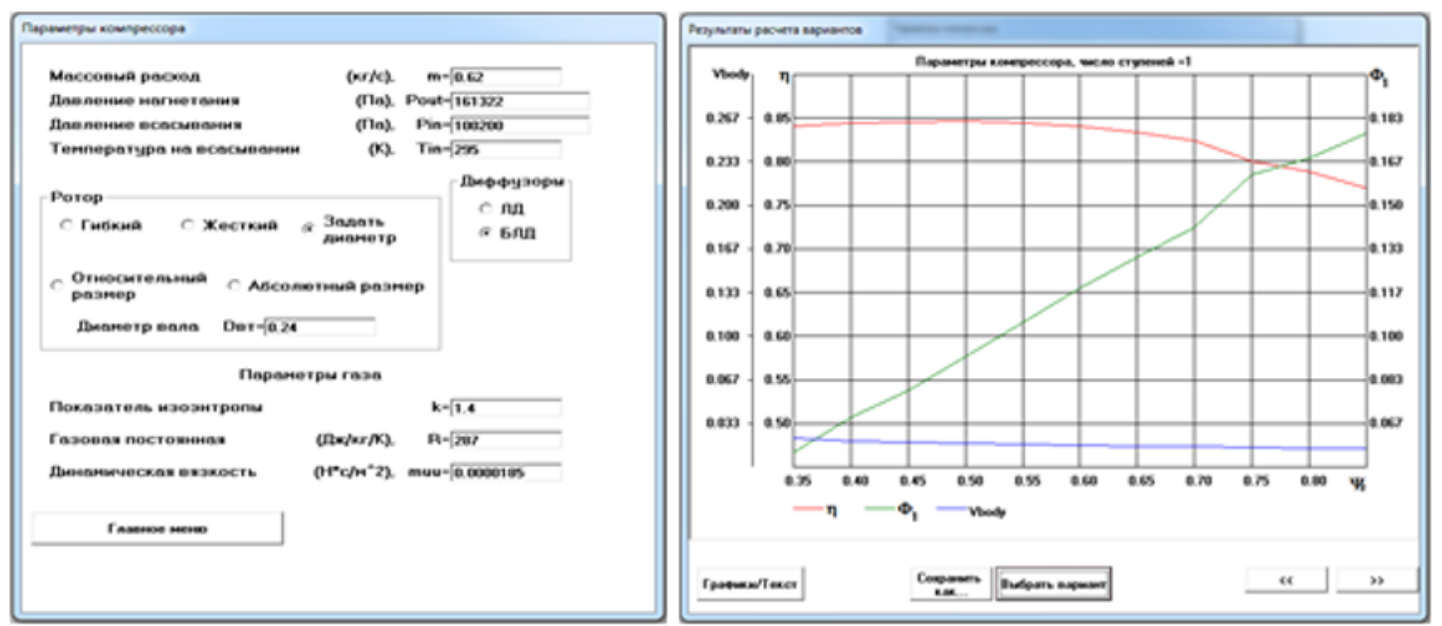

Fig. 3. The program of single-shaft centrifugal compressor candidates' comparison and preliminary design (SSCCPD). On the left - the compressor parameters. On the right - candidates' efficiency, flow rate and the approximate case volume versus the loading factor. 
Table 1. SSCCPD program. Part of the output file of the candidates' calculation. 140E compressor variant selected for the design.

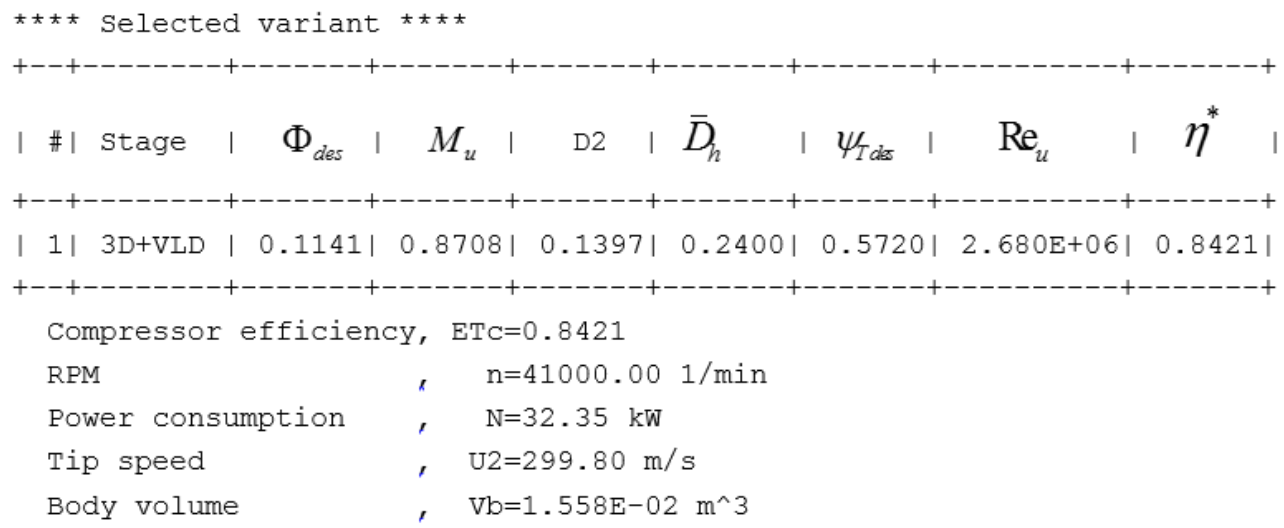

inlet).

From Figure 3 it follows that a simplified mathematical model to obtain maximum efficiency recommends choosing the candidate with $\psi_{\text {Tdes }}=0.50$ with the blade velocity $325 \mathrm{~m} / \mathrm{s}$ and the impeller diameter being $151 \mathrm{~mm}$. The expected efficiency is 0.847 .

For the final design, a candidate with a slightly larger loading factor $\psi_{\text {Tdes }}=0.572$ is taken. Its parameters are presented in Table 1 . The selected compressor variant has a blade velocity of $300 \mathrm{~m} / \mathrm{s}$, impeller diameter 140 $\mathrm{mm}$, but the efficiency is lower by $0.49 \%$. Despite this, for a medium-speed engine with a very long service life, an impeller with a lower blade velocity is preferable. The designed compressor is named 140E. The turbocharger with this compressor is named TKR 140E.

In the Table 1: $M_{u}=u_{2} / \sqrt{k R T_{i n l}^{*}}$ is the blade's Mach number; $\quad \bar{D}_{h}=D_{h} / D_{2}$ is hub ratio; $k$ is isentipic coefficient; $R$ is gas constant; $T_{i n l}^{*}$ is total temperature at the inlet.

\section{Preliminary design}

After the preferred compressor candidate is selected, a preliminary design program calculates all the dimensions necessary for characteristics calculating. The dimensions are automatically transferred to the program for characteristics calculating of a single-stage compressor (OPTIM 2 program).

Compressor TKR 130 stator elements were offered by NPO "Turbotechnica" in accordance with their design method. According to the primary design, the stator element's dimensions are close to the dimensions offered by NPO "Turbotechnica".

In the preliminary design, the dimensions of the impeller blades are also calculated. This allows to check the compliance of the impeller preliminary design with the technical specification. Figure 4 shows velocity diagrams of an inviscid flow on the preliminary designed impeller blades. Calculations of an inviscid quasi-threedimensional flow are carried out according to the program 3DM.023 [13].

The flow character corresponds to the recommendations in the monograph [13], but at the shroud blade-to-blade surface, the velocity peak at the leading edge indicates a negative incidence angle. Not optimally large deceleration of the relative velocity is noticeable. Flow deceleration on the blades suction side on the shroud blade-to-blade surface should also be reduced.
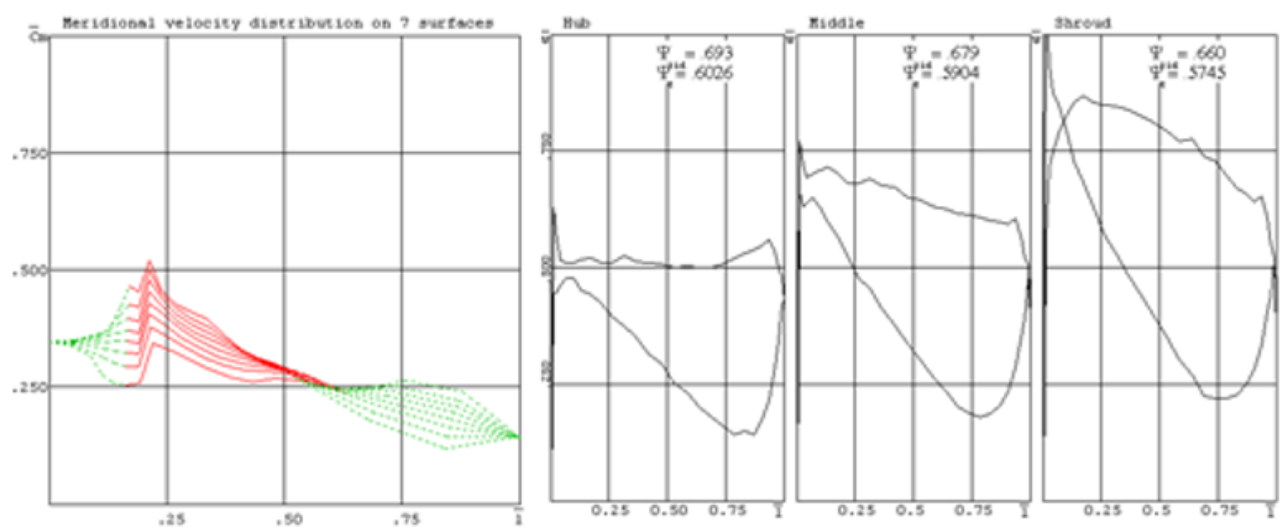

Fig. 4. 3DM.023 program. The primary design of the compressor 140E impeller. On the left are the meridional velocities on eight blade-to-blade surfaces, on the right are the velocity diagrams on the three blade-to-blade surface. 

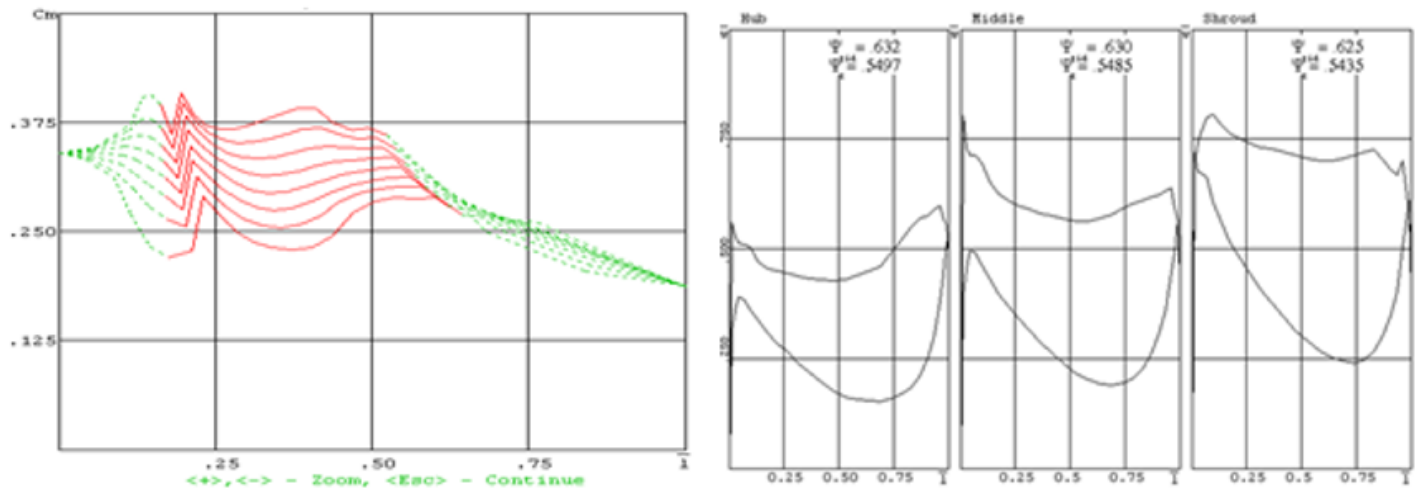

Fig. 5. 3DM.023 program. Final design of the compressor 140E impeller. On the left are the meridional velocities on eight bladeto-blade surfaces, on the right the velocity diagrams on the three blade-to-blade surface.

\section{Final design TKR 140E}

The quasi-three dimensional inviscid flow calculation using the 3DM.023 PC software presents the value of the loading factor $\psi_{\text {Tid }}$. Viscosity reduces the loading factor, which is taken into account by the empirical coefficient $K_{\mu}=\psi_{\text {Tdes }} / \psi_{\text {Tid }}$. The value adopted at the preliminary design $K_{\mu}=0.92$ is based on experiments with stages with return channels. Turbocharger TKR stages include a scroll. In this stage, the flow is characterized by a circumferentially uneven pressure field, which creates a non-stationarity of the flow in the impeller. The test data of the NPO "Turbotekhnika" were reduced. The value of empirical coefficient is $K_{\mu}=$ 0.98 for the impellers similar to $140 \mathrm{E}$.

The value of the loading factor of the impeller $140 \mathrm{E}$ $\psi_{T \text { des }}=0.585$ is $2 \%$ more than that of the adopted candidate (Table 1). This is a common design practice. The well-known European standard [0] requires unconditional provision of a design pressure ratio, allowing an excess of power consumption up to $4 \%$. In order to obtain the desired value $\psi_{T \text { id }}=\psi_{\text {Tdes }} / K_{\mu}=$ $0.585 / 0.98=0.597$ with non-incidence flow on shroud and to optimize the velocity diagrams the variants of the blade row were considered.

The form of the function $\beta_{b l s}=f\left(\bar{l}_{m}\right)$ (where $\beta_{b l s}$ is the impeller blade angle on shroud; $\bar{l}_{m}$ is the impeller blade relative length in meridional plane) with double curvature is important. This form is explained by the desire to eliminate the "saddle" in the velocity diagram on the shroud current line. The velocity diagrams are shown in Figure 5 on the right.

A complete elimination of the "saddle", i.e. to obtain a linear decrease in velocity along the blade suction side $\bar{w}_{s}$ (where $\bar{w}_{s}$ is relative velocity at the flow separation point on the blade profile) was not possible. In accordance with the recommendations [0-0], an increase in the exit blade angle from the hub to the shroud is taken. The diagrams of meridional velocities are shown in Figure 5 on the left.

Figure 6 shows the 140E impeller blade row according to the data of the 3DM.023 software and the view of the solid model calculated by the "3DCompressor" program included in the system of the endto-end design programs. The software reads the dimensions obtained as a result of the design using the Universal modelling method and transfers the information to the commercial package SolidWorks,

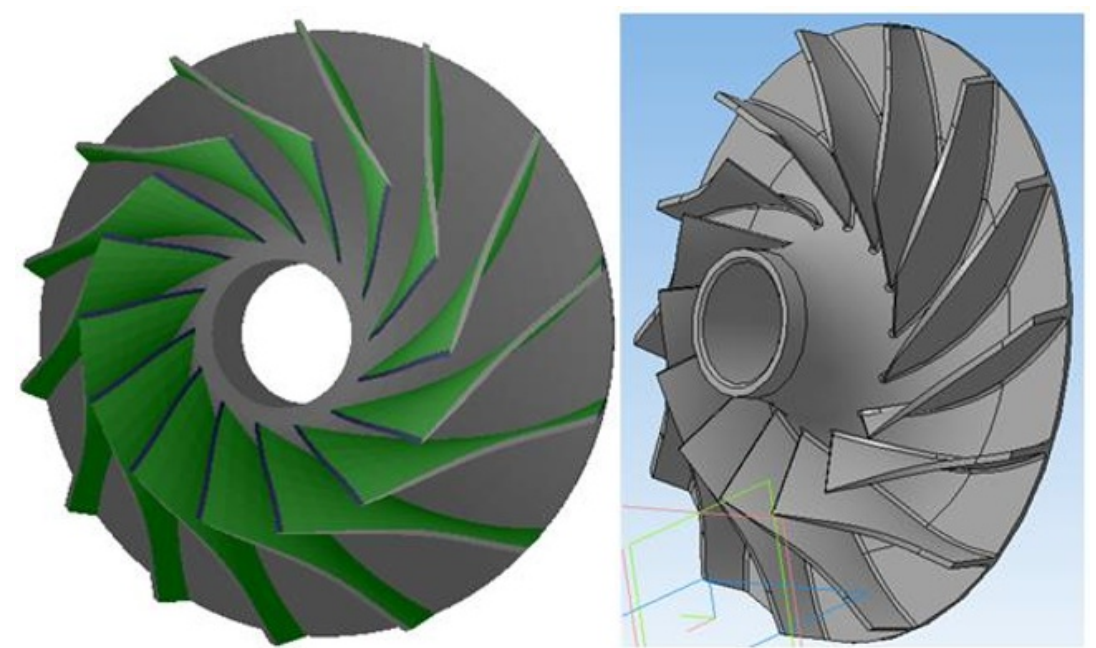

Fig. 6. TKR 140E impeller blade row view. On the left are results obtained with the 3DM.023 software. On the right is a view of a solid model obtained using the SolidWorks software. 
which automatically builds a 3D model.

A family of $140 \mathrm{E}$ compressor characteristics is calculated $\eta^{*}, \pi^{*}, N=f(\bar{m})$ (where $N$ is shaft power; $\eta^{*}$ is polytrophic efficiency) at blade velocities 150 , 200, 250 and $300 \mathrm{~m} / \mathrm{s}$ using the direct gas dynamic solution software. The characteristics are presented below in comparison with the test results.

\section{Test results. Comparison with the project}

Figure 7 shows a section of the turbocharger TKR 140E of NPO "Turbotekhnika". The measured and calculated characteristics of the $140 \mathrm{E}$ compressor are compared on Figure 8.

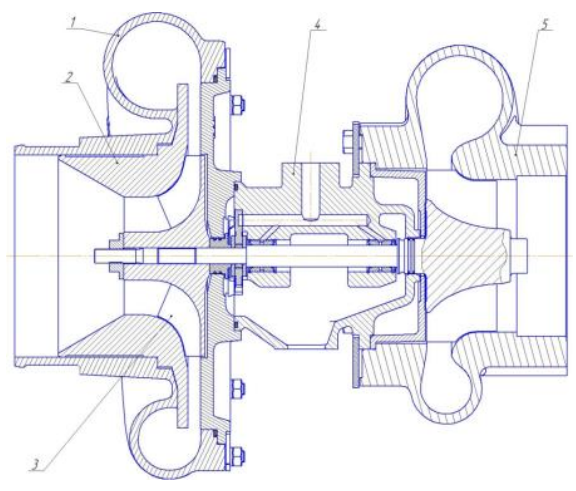

Fig. 7. Sectional drawing of turbocharger TKR 140E. $1-$ compressor housing; 2 - insert; 3 - impeller (R\&D laboratory "Gas dynamic of turbomachines" project); 4 - bearing housing; 5 - turbine.

At the design flow rate $\bar{m}_{\text {des }}=0.62 \mathrm{~kg} / \mathrm{s}$ at $u_{2}=300$ $\mathrm{m} / \mathrm{s}$ the specified pressure ratio is ensured almost

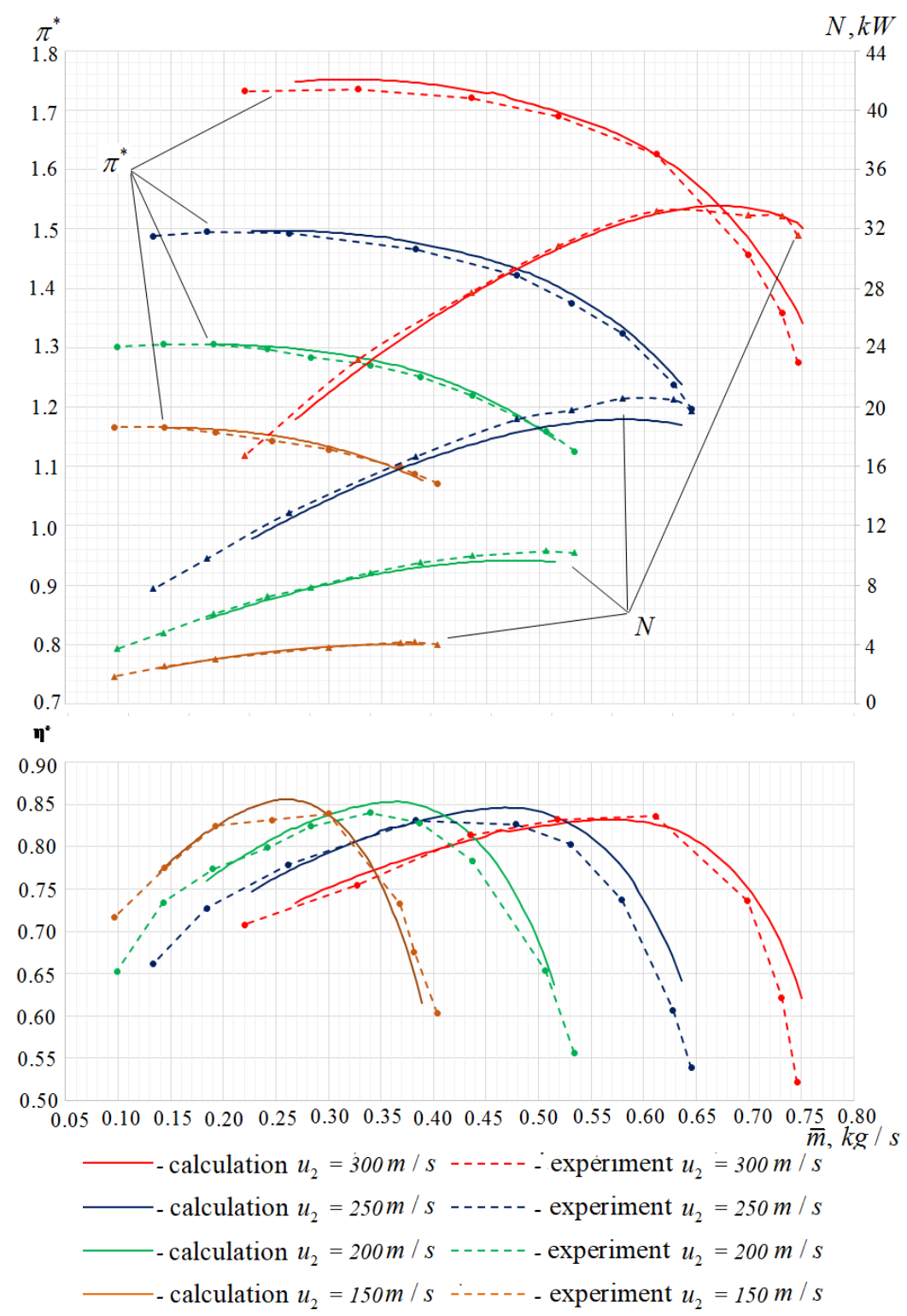

Fig. 8 Comparison of design and experimental characteristics of model 140E compressor at blade velocities $u_{2}=150,200,250$ and $300 \mathrm{~m} / \mathrm{s}$. 
exactly. The expected efficiency is confirmed. The mathematical model overestimates the efficiency and pressure ratio in off-design modes. At the same time, the calculated maximum pressure ratio flow rate is calculated closer to the design flow rate in comparison with the experiment. As experimental data accumulate, the empirical coefficients of the mathematical model will be refined.

\section{Conclusion}

The new version of the Universal modeling method has confirmed the effectiveness with regard to the design of relatively small-sized turbochargers compressors.

The compressor $140 \mathrm{E}$ has good gas-dynamic characteristics. This design method can be applied for other turbocharger deigns.

\section{Acknowledgements}

The research was performed by a Grant of the President of the Russian Federation for young $\mathrm{PhD}$ MK1893.2020.8.

\section{References}

[1] V.E. Evdokimov, Turbines and compressors 3, 4 (1997)

[2] A.T. Lunev, Compressors and pneumatics 10, 4-7 (2001)

[3] K. Kabalyk, W. Kryłłowicz, Transactions of the institute of fluid-flow machinery 131, 41-53 (2016)

[4] W. Kryłłowicz, P. Świder, Z. Kozanecki, K. Kabalyk, Jr.Z. Kozanecki, 12th European Conference on Turbomachinery Fluid Dynamics and Thermodynamics, 127934 (2017)

[5] V. Melnikov, A. Proskurov, CAD and graphics 4, 92-96 (2005)

[6] K. Lusardi, Thesis for the Degree of Master of Science, 90 (2012)

[7] Y. Galerkin, Proceedings of the science school of compressor engineering SPbGPU (M.: pub. SPbGPU, 2010)

[8] A. Drozdov, Y. Galerkin, AIP Conf. Proc. 030052-1-030052-10 (2018)

[9] Y. Galerkin, K. Soldatova, A. Drozdov, A. Rekstin, ASME 2015 Gas Turbine India Conference, 119665 (2015)

[10] A. Rekstin, Y. Popova, A. Ucehovscy, AIP Conf. Proc., 030036-1-030036-11 (2018)

[11] A. Rekstin, K. Soldatova, Y. Galerkin, University news 9, 702 (2018)

[12] A. Rekstin, B.V. Bakaev, Scientific and technical statements SPbPU 24, 4, 24-38 (2018)

[13] Y. Galerkin, Turbocompressors (Information and publishing center KHT, 596, 2010)
[14] ISO 5389 Turbocompressors Performance test code (1992)

[15] Y. Galerkin, A. Drozdov, ASME 2015 Gas Turbine India Conference, 119665 (2015)

[16] Y. Galerkin, A. Drozdov, Scientific and technical statements SPbPU 4, 231, 179-188 (2015) 